\title{
Expressing our Uniqueness to Create a Sustainable Way of Life
}

\author{
Dott.ssa Elena DDV Dragotto ${ }^{1}$
}

\begin{abstract}
In a society where points of reference and specific roles are also lacking in human relations, where self-expression is often confused with unattainable, false, alien and frustrating stereotypes, developing a personal growth process that leads one to recognize one's Uniqueness, allows one to build more intimate and satisfying relationships with oneself and with others.

The result is the development of a society in which everyone perceives their own value, regardless of fashions and external models, and everyone is aware of their role and responsibilities, contributing with their Uniqueness to the development of their own complexity and richness.

The approach of Voice Dialogue Counseling, created in the 70's by psychologists and psychotherapists $\mathrm{Hal}$ and Sidra Stone, has proven to be very effective in helping people to discover and develop their own Uniqueness.

With great respect for everyone's human experience, Voice Dialogue Counseling aids people in expanding themselves, embracing more and more of their richness and complexity, without any judgment.

Supporting human Uniqueness means safeguarding a valuable resource and making it available for the development of Humanity and the planet.
\end{abstract}

Key words: Uniqueness, counseling, Voice Dialogue, Psychology of Selves, buman relations, personal growth, Gross National Happiness

\section{Introduction}

In today's society characterized by great change and evolution, there is an absence of those points of reference and those defined roles which allow people to affirm a reassuring identify and to develop relationships that are comforting, stable and lasting.

One of the consequences of this era, born to counter the great vulnerability of modern societies and individuals that has come about, is the necessity "to be visible", a need that is supported by the prevailing culture - from the development of Social Media to the numerous "Talent Shows" that flood the television. Unfortunately, quite often, this "sine qua non" condition of visibility at all costs is confused with freedom and the natural human need for self-expression. This misunderstanding has painful and damaging consequences both for the individual and the society at large.

In fact, the exasperating effort to imitate unattainable and alien stereotypes has the natural effect of creating frustration, regret, feelings of emptiness and uselessness that hinder the perception of the sense of one's own life and that cloud the vision of one's own future. Individuals that are unfulfilled, frustrated and unaware of their own value, 
are a high price to society in terms of shared wellbeing, sustainable and satisfying human relationships, creativity, responsibility and health.

A society and a culture that encourage actions that have their roots in the desire to imitate others with the goal of being "better than" or "more than" rather than following one's unique, personal inner needs, and that support competition in order to earn a visibility irrespective of one's true interests and wishes, these are destined to fail to produce the real effects of evolution, wellbeing and true encounters with others.

Thus, in order to develop sustainable relationships and to truly live, more than ever before it appears to be necessary to identify, support, and provide inner and outer spaces, reflection, visions, and models that sustain individuals in the discovery and the recognition of their Uniqueness.

The recognition on the part of human beings of their personal Uniqueness, has, as a natural consequence, the development of a society in which every individual perceives their own personal worth regardless of fashions or external models, and is conscious of and responsible for their own role, contributing to the development of their own complexity/richness.

A real opportunity to reach this goal is offered by counseling, a relatively young profession here in Italy, but with a longer history in the Anglo-Saxon world where it was born. Of particular interest, in this regard, is the Voice Dialogue relational counseling.

\section{Counseling}

”. (Rollo May)

"... the function of the counselor is to belp the client to become what he was destined to be ...

The counselor (or counsellor for the English), was born as a genuine professional title in the United States in the 1920's, to identify those people who although they carried out jobs that require a thorough knowledge of the human personality, were neither psychologists or psychotherapists. In Europe, counseling (or counselling) developed in the 1930's, starting in Great Britain and in Italy it exists as a discipline in itself since the 1980's.

The term "Counseling" is used to describe a helping relationship that exists between two people in which one turns to the other in response to a specific need related to the family environment, emotional relationships, the work place or for self-realization.

In particular, Relational Counseling "is a professional activity aimed at improving the relational wellbeing of people and a heightened quality of life through the development of a greater interpersonal awareness as well as relational-communication skills and abilities.

These skills allow individuals to manage effectively interpersonal communication and relational conflicts.". 1

Its characteristic of being an activity that empowers individuals and the collective places it squarely within Humanistic culture as a necessary and essential element to create individuals that are conscious, deep, responsible, and respectful of others. It also

1

Associazione Nazionale Counselor Relazionali (National Association of Relational Counselors). Chi è il counselor relazionale (What is a relational counselor?). http://www.ancore.org/counseling/il-counselingrelazionale.html 
effectively meets the requirements of the current need within individuals and society at large to develop a vision in which men and women are the makers and the masters of their own destiny, each with their own diversity and innumerable creative capacities. This is all possible thanks to the fact that counseling activates processes of personal growth and improvement, allowing individuals to find areas for personal and collective expression and it educates them in the freedom of being themselves.

\section{Voice Dialogue}

"Accordingly it is not surprising that some people have great resistance to seeing their personality as other than one monolithic, coherent unit. Something inside them resists the awareness that their personality is made up of many different parts - as if to admit such a breakdown would cause the breaking down into non-being of their identity itself.

More often, once we become aware of the diversity of elements in our personality, we accept them in principle, but continue to reject them operationally, in practice. As we live our lives, we continue thinking about ourselves and about others as if we were made "all in one piece," as if we were already whole. We seldom think of ourselves - and of others - as made up of different parts. We sort of know it in theory, but in practice forget about it. So if we are talking to somebody who's being obnoxious, we say, 'He's obnoxious," and may get angry at him. And if an hour later he becomes happy and cheery, we say, "Well, he changed, he's almost like a different person". ${ }^{2}$

Voice Dialogue is a sophisticated technique of "interview of the Selves" that aims at developing an "Aware Ego" process that, through the development of freedom of choice, allows individuals to decide in a conscious way, in each situation, what inner aspects to activate.

The Psychology of Selves and Voice Dialogue were born in the United States in the 1970's, conceived by Hal Stone, Ph.D. and Sidra Stone, Ph.D., and were founded on the observation that the Ego is composed of a multiplicity of Inner Selves.

"The Psychology of Selves provides a clear explanation of how these selves operate in your life and how they keep you from realizing your full potential. Learn how these selves determine the way you see the world, control your behavior, and limit your choices. Most of us live a much smaller life than necessary. Learn to be more than any one of your individual selves". (Hal and Sidra Stone)

When we are born, we are completely vulnerable, our survival depends exclusively on the care of our parents or of other care-givers. We also have at our complete disposal a wealth of potential behaviors that we can use during our life: there are no limits, the baggage of every human being corresponds to all the behaviors that exist in the world, from those that are the most abhorrent to those that are the most edifying, each one of us possesses them all. So, what happens in the first years of our life?

With this immense wealth of possibilities, we begin to make a selection and the choices are made based on the consequences of the behaviors acted out.

The innate needs of belonging and being loved, push human beings to favor those behaviors, those values, those points of view that safeguard our needs. As a result, even though the process is unconscious, individuals will make a choice and identify with those

2 SUBPERSONALITIES, James Vargiu, Synthesis Volume I: The Realization of the Self. The Synthesis Press, Redwood City, CA 1974 
Selves, the Primary Selves, that permit them to satisfy the needs that are ancestrally linked to survival. In the moment in which one identifies with a group of Selves, the Operating Ego, even if this takes places totally unconsciously, automatically its opposites, the Disowned Selves, are pushed into the Unconscious. Thus, this immense potential and the richness of which human beings are bearers quickly is reduced to a few, repetitive behaviors that do not allow for a true expression of the self and freedom in living.

\section{Voice Dialogue relational counseling}

The objectives of Voice Dialogue relational counseling are the dis-identification from the Primary Selves in which the individual identifies; the welcoming of the Disowned Selves into one's life with the resulting broadening of oneself and the expansion of one's inner resources; the taking charge of one's vulnerability; the activation of the Aware Ego process that allows for free and conscious choices.

In general, clients have completed a cycle of 8/10 sessions of individual Voice Dialogue relational counseling of one hour length, once a week or once every fifteen days; or group sessions during workshops or training of varying lengths. In both situations, it was possible to observe that in all cases the counseling sessions, by activating the Aware Ego process,

enabled the clients to harbor their own internal riches and activate a process of empowerment, both essential objectives in order to recognize one's Uniqueness, to afford it attention and to have the courage to pursue it in one's own life.

In fact, through Voice Dialogue relational counseling it was possible for clients to learn that their habitual behaviors are acted out, in the majority of the cases, in accordance with some parts of themselves, the Primary Selves. This awareness, has allowed them to stop taking their life for granted as the only one possible and to realize that their actions were not truly inspired and guided by their deepest and most intimate feelings in accordance with their own Uniqueness.

With great respect for the human experience of each individual, Voice Dialogue counseling has helped people to widen themselves and to embrace ever more deeply, without judgment, the richness and complexity that each one possesses.

The separation from the Primary Selves allows the clients to open themselves to other possible ways of acting and of looking at the world and at life. It is exactly this process that naturally brings to light one's Uniqueness. When one's actions are no longer "automatic" because they are guided by the Primary Selves but instead are free actions inspired by the Aware Ego, what emerges ever more frequently over time is a feeling of authenticity in one's actions and way of being, which leads finally to the recognition of one's Uniqueness of expression and of being.

A fundamental and crucial point in the course of Voice Dialogue counseling that has allowed clients to let go of the need to "be special" in favor of being "Unique" has been to confront one's vulnerability. It is at this point that one experiences the greatest change, passing from the request for attention from outside to the taking charge of oneself.

The Aware Ego process that was activated in the counseling sessions had allowed clients 
to develop a sense of responsibility with regard to themselves and their primary needs, and thus freed themselves from a way of acting that was permeated with the need to be heard, loved and seen by others.

In summary, during Voice Dialogue relational counseling, both with individuals and groups, the following effects had been observed in each of the participants:

- $\quad$ Empowerment;

- $\quad$ Growth of self-esteem;

- Recognition/acknowledgement of one's Worth

- Development of free will;

- Increased creativity;

- Growth of responsibility for oneself in relationships and in one's actions;

- Development of compassion and decrease in judgment and feelings of separation;

- $\quad$ Respect for oneself and others;

- More intimate and satisfying relationships;

- Increase in the variety of approaches available to deal with life's challenges.

In conclusion, in the course of Voice Dialogue relational counseling a fundamental transformation had taken place: from living life with the need to be "Special", to the recognition and respect for one's Uniqueness.

\section{Uniqueness: a Sustainable Way of Life}

"Furthermore, you are a one-of-a-kind miracle. Let's consider the evidence: each fingerprint on every human being is different. Imagine, four billion people now present in the world, plus all those who have come before and will come in the future. All have their own unique fingerprints. There are no duplicates. [...] And yet it is an indisputable fact. Each of us is different'. 3

We have seen that in a society where in order to be valued, loved, esteemed, one needs to be "Special", it is not easy to be oneself. Daily life is more complex than it used to be, but neither the family, nor the school, nor other institutions are concerned with developing an emotional intelligence capable of dealing with this complexity. Thus, where there is fragility/vulnerability, the natural tendency is to protect it, not to listen to it, to find a way to not feel insecure, inadequate, unsuitable or incapable. The main cause of this common malaise is one of the inner Selves that is the most difficult to manage, and one that is reinforced by the dominant culture, the Inner Critic.

The Inner Critic is that part of ourselves that criticizes and judges the thoughts, actions and feelings of everyone: that utilizes the comparison with the world outside to make people feel inadequate, inferior, "less than". Most people are unaware of its activity and its role in their inner dynamics.

Driven by the Inner Critic, which is amplified by a society and a culture that requires "visibility through performance" in order to have status and value, people prematurely

\section{${ }^{3}$ Your Many Faces: The First Step to Being Loved, Virginia Satir, Celestial Arts, Berkeley CA 2009}


distance themselves from their "own unique fingerprint", in order to embrace models and values that, more often than not, do not correspond thoroughly and intimately to their own, but guarantee that social identity and visibility that allows them to feel adequate and accepted.

On the other hand, it is just this sense of inadequacy that is the target of the majority of advertisements, creating models of people that are always more impeccable, young, healthy, thin, beautiful, good-smelling, intelligent, productive, rich.

It is inevitable that this confronts people with the frustration of never being enough, neither for one's own Inner Critic, nor for the society and culture in which one lives. Being oneself is definitely not in fashion!

This kind of culture and form of education that has as an objective "being Special" renders "unsustainable" the condition of a human being eager to express himself and his potential. To be Special is exhausting and painful.

It is exhausting because it forces a person to always be "more than the others", to compete, to avoid others in order to avoid discovering that perhaps the other is better than he is. It is painful because "to be Special" means to renounce one's dreams and desires, in the last analysis oneself, in exchange for being loved at all costs.

It is one's Uniqueness that is the true alternative, and it is the search for this Uniqueness on the part of the individual that helps to build the wellbeing of a society and of the individuals that form it: the Gross National Happiness (GNH). Or perhaps it should be re-baptized the Gross National Uniqueness (GNU).

The process of counseling, and in particular Voice Dialogue relational counseling, has helped people to recognize their own Worth, identify their own resources and talents, their own Uniqueness.

This is because it is one's Uniqueness that gives us a place in the world and reassures us of the value of who we are. Acting from one's Uniqueness guarantees success, it is fulfilling, heady, it gives Humanity the possibility of using all the riches of which each one of us is the bearer. Being faithful to one's Uniqueness opens up the possibility of truly intimate relationships and partnership with creativity, with the development of networks that integrate many fields of knowledge; with empathy, with the logic of a winwin situation, and with sharing.

\section{Conclusion}

The respect for the riches of each human being has the same value as the respect that we should have for the Planet that hosts us and bestows upon us so unconditionally its riches. To promote, through focused efforts, individual expression that is deep and unique is an endeavor that has enormous repercussions for the quality of life and the level of respect for all that surrounds us.

For this reason, counseling, which does not focus on pathology but rather on the wellbeing of human beings, is an exceptional tool in this era of great change because it responds to new instances of complexity, unpredictability, vulnerability and the new. In particular, Voice Dialogue relational counseling has provided people with instruments to "flow" with the complexity of modern life, teaching them to manage first and foremost their inner complexity without either judging or renouncing any part of themselves. 
In conclusion, we believe that to support the development of the Uniqueness of human beings means to safeguard a valuable resource and to make it available for a sustainment development of Humanity and the Planet.

\section{References}

Budde, J.; Collewijn, B. (2013). Me, my selves and I. Uitgeverij Thema

Dragotto, E. (2003). "Il conflitto esterno specchio del conflitto interno: Il dialogo delle voci interiori", in La comunicazione come antidoto ai conflitti a cura di E. Cheli, Punto di fuga editore. http://www3.unisi.it/mastercomrel/articoli $\% 20 \mathrm{e} \% 20$ saggi/art.dragotto.htm

Dyak, M., (1999). The Voice Dialogue Facilitator's Handbook, Part 1: A Step-by-Step Guide to Working with the Aware Ego, Novato, New World Libray

Errani Civita, F. "Voice Dialogue: alla scoperta del nostro mondo interiore"; http://www.corem.unisi.it/bibliografia/articoli/errani_civita-voice_dialogue.pdf

Neukrug, E., S., (2015). The SAGE Encyclopedia of Theory in Counseling and Psychotherapy, SAGE Publications, Inc (Voice Dialogue counseling)

Stamboliev, R. "Coaching with Polarities. Introducing the Stretching technique", in International Journal for Mentoring and Coaching, XII, $n^{\circ}$ 1, 2014, pp. 66-76.

Schwartz, R. "Our multiple selves" - Family therapy networker, 1987. http://www.hakomiinstitute.com/Forum/Issue10/OurMultipleSelves.pdf

Stone, H., Stone, S., (1993). Embracing Our Selves: The Voice Dialogue Manual. Nataraj Publishing

Stone, H., Stone, S., (1993) Embracing Your Inner Critic: Turning Self-Criticism into a Creative Asset. San Francisco: HarperSanFrancisco

Stone, H., Stone, S., (1989). Embracing Each Other — Relationship As Teacher, Healer \& Guide. Delos, Inc., Albion, California

Stone H., Stone S. Principi e metodo del voice dialogue, http://www3.unisi.it/mastercomrel/articoli $\% 20 \mathrm{e} \% 20$ saggi/principi\%20voice.htm

Stone H., Stone S. Voice Dialogue: An Introduction to the Use of Voice Dialogue, http://voicedialogue.org/articles/Voice_Dialogue-_An_Introduction.htm

Stone H., Stone S. The Basic Elements of Voice Dialogue, Relationship, and the Psychology of Selves, their origins and development. http://www.voicedialogueinternational.com/pdf/Elements.pdf

Vargiu,J.G. (1974) SUBPERSONALITIES, Synthesis Volume I: The Realization of the Self. The Synthesis Press, Redwood City, CA http://www.synthesiscenter.org/articles/1660.pdf

Vargiu, J. G. (1974). The theory: Subpersonalities. Institute of Psychosynthesis. 